\title{
Therapeutic Potential of Human Turbinate-Derived Mesenchymal Stem Cells in Experimental Acute Ischemic Stroke
}

\author{
Hyosun Lim¹, Sun Hwa Park², Sung Won Kim², Kyung-Ok Cho ${ }^{1}$ \\ ${ }^{1}$ Department of Pharmacology, Department of Biomedicine \& Health Sciences, Catholic Neuroscience Institute, College of Medicine, The Catholic University \\ of Korea, Seoul, Korea \\ ${ }^{2}$ Department of Otolaryngology-Head and Neck Surgery, College of Medicine, The Catholic University of Korea, Seoul, Korea
}

\begin{abstract}
Purpose: Mesenchymal stem cells (MSCs) have demonstrated great promises for the treatment of ischemic stroke. Previously, we identified a new source of MSCs located in the inferior turbinate. We investigated therapeutic potentials of human turbinate-derived mesenchymal stem cells (hTMSCs) in ischemic stroke.

Methods: Ischemic stroke was induced by the intraluminal occlusion of middle cerebral artery (MCAo) for 50 minutes in rats. At one day after MCAo, hTMSCs, adipose tissue-derived MSCs (AdMSCs), or phosphate buffered saline (PBS) were transplanted into the striatum. Functional recovery was assessed by repeating behavioral tests including modified neurologic severity score and corner test. At 14 days after MCAo, brains were stained with hematoxylin and eosin (H\&E) for measuring infarct volume. The survival of grafted MSCs was evaluated by immunohistochemistry to human nuclei (hNU). Immunohistochemistry with anti-doublecortin (anti-DCX) was performed to assess hippocampal neurogenesis.

Results: Transplantation of hTMSCs following MCAo showed improvements of neurologic function, which was comparable with that of AdMSCs. H\&E staining showed no difference in infarct volume among 3 groups. Regarding the survival of grafted MSCs, the number of hNU-expressing cells was not different between hTMSCs- and AdMSCs-treated groups. Finally, hTMSCs increased the number of subgranular DCX-positive cells compared to PBS-treated controls, without affecting hilar ectopic migration of newborn neurons.

Conclusions: hTMSCs could improve functional recovery following ischemic stroke, of which efficacy was similar to AdMSCs. Although hTMSCs showed comparable infarct size and survival of grafted MSCs, transplantation of hTMSCs could upregulate subgranular neurogenesis with no impact on ectopically migrating newborn neurons.
\end{abstract}

Keywords: Mesenchymal stem cells; Transplantation; Stroke; Turbinates; Neurogenesis; Rats

- Fund/Grant Support: This work was supported by National Research Foundation of Korea (NRF) grants funded by the Korean government (NRF-2017R1D1A1B03029812) and by the Korea Health Technology R\&D Project through the Korea Health Industry Development Institute (KHIDI) funded by the Ministry of Health \& Welfare (HI15C2854).

- Research Ethics: All animal studies were performed according to the Ethics Committee of The Catholic University of Korea and were carried out in accordance with the National Institutes of Health Guide for the Care and Use of Laboratory Animals (NIH Publication No. 80-23, revised 1996). Isolation of turbinate-derived human mesenchymal stem cells was approved by the Institutional Review Board of The Catholic University of Korea.

- Conflict of Interest: No potential conflict of interest relevant to this article was reported.

\section{- HIGHLIGHTS}

- Transplantation of hTMSCs promoted functional recovery following ischemic stroke.

- Neuroprotective effects and the survival of grafted MSCs did not differ between hTMSCs and AdMSCs.

- Transplantation of hTMSCs after stroke enhanced dentate neurogenesis, without affecting aberrant hippocampal neurogenesis.

Corresponding author: Kyung-Ok Cho (iD http://orcid.org/0000-0002-1314-7584 Department of Pharmacology, College of Medicine, The Catholic University of Korea, 222 Banpo-daero, Seocho-gu, Seoul 06591, Korea

E-mail: kocho@catholic.ac.kr /Tel: +82-2-2258-7329 / Fax: +82-2-536-2485

Submitted: September 12, 2018 / Accepted after revision: October 8, 2018
This is an Open Access article distributed under the terms of the Creative Commons Attribution Non-Commercial License (http://creativecommons.org/licenses/by-nc/4.0/) which permits unrestricted non-commercial use, distribution, and reproduction in any medium, provided the original work is properly cited. 


\section{INTRODUCTION}

Stroke has been the second highest cause of death and longterm disability worldwide for the last 15 years, approximately accounting for 5.78 million deaths in 2016 [1]. Ischemic stroke where cerebral blood vessels are occluded by thrombi or emboli is more prevalent than the hemorrhagic stroke caused by parenchymal or subarachnoid hemorrhages [2]. Guidelines recommended for the treatment of ischemic stroke are either administration of intravenous recombinant tissue plasminogen activator within 4.5-hour window from symptom onset or endovascular procedures recanalizing the obstructed vessels [3]. However, in spite of significant advancements in medical care and biological researches, there are still no cures for the ischemic stroke and the time constraint issue of current acute stroke therapies required developments of novel therapeutic strategies.

Cell therapy has become one of the promising approaches for treating ischemic stroke in experimental rodent settings and human patients [4-6]. Among various sources of stem cells, transplantation of mesenchymal stem cells (MSCs) has been reported to improve neurological function following stroke [7]. MSCs are multipotent adult stem cells that can maintain multilineage differentiation potential and self-renewal, in addition to low immunogenicity following transplantation $[8,9]$. Ever since MSCs originated from bone marrow have demonstrated therapeutic efficacy against ischemic stroke [10,11], diverse tissues compartments including adipose tissue, skin, placenta, liver, and lung have been found to harbor MSCs [9]. In addition, we have identified a new source of MSCs that was isolated from the inferior turbinate in humans with nasal septal deviation [12-14]. Human turbinate-derived MSCs (hTMSCs) can show stable proliferative activities with the expression of stem cell markers and they can differentiate into osteocyte, adipocyte, and chondrocytes [12,13], suggesting a new functional multipotent MSCs. Therefore, in the present study, we investigated therapeutic potentials of hTMSCs in a rat model of ischemic stroke and compared the efficacy of hTMSCs with adipose tissue-derived MSCs (AdMSCs), a well-known type of MSCs that already showed neurologic benefits following ischemic stroke [15]. We then assessed the infarct size, and the survival of grafted hTMSCs and AdMSCs in the ischemic brain tissues after cell transplantation. Finally, we demonstrated increased hippocampal neurogenesis by the administration of hTMSCs, providing a possible mechanism underlying beneficial effects of hTMSCs after ischemic stroke.

\section{MATERIALS AND METHODS}

\section{Preparation of hTMSCs and AdMSCs}

Human MSCs were isolated from waste nasal inferior turbinate and adipose tissues during surgery with the approval of the Institutional Review Board of The Catholic University of Korea (KC08TISS0341, MC16CISI0087, respectively). At passage 4, hTMSCs and AdMSCs were grown in Alpha minimum essential medium (Alpha MEM) (Welgene, Gyeongsan, Korea) or low glucose Dulbecco's modified Eagle's medium (Hyclon, GE Healthcare Life Sciences, Little Chalfont, UK), respectively, supplemented with $10 \%$ fetal bovine serum, penicillin and streptomycin at $37^{\circ} \mathrm{C}$.

\section{Rat Model of Transient Focal Cerebral Ischemia and Transplantation of hTMSCs or AdMSCs}

All experimental procedures were carried out according to the guidelines of the Institutional Animal Care and Use Committee of The Catholic University of Korea and the National Institutes of Health Guide for the Care and Use of Laboratory Animals (NIH Publications No. 80-23). Transient focal cerebral ischemia was induced by the intraluminal occlusion of middle cerebral artery (MCAo) as previously described with minor modifications [16]. Briefly, Sprague-Dawley male rats, weighing 270$300 \mathrm{~g}$, were anesthetized with $1.5 \%$ isoflurane in a $70 \%$ nitrous oxide and $30 \%$ oxygen mixture using a face mask. After midline neck incision, a 5-0 silicon-coated nylon monofilament (Doccol Corp., Sharon, MA, USA) was inserted through the external carotid artery and into the internal carotid artery. At 50 minutes after the left MCAo, the filament was withdrawn to allow reperfusion and animals were placed in the incubator. During cerebral ischemia, rectal temperature was maintained at $37^{\circ} \mathrm{C} \pm 0.5^{\circ} \mathrm{C}$ using a thermistor-controlled heating blanket. At one day after MCAo, rats were reanesthetized with ketamine $(100 \mathrm{mg} / \mathrm{kg})$ and xylazine $(10 \mathrm{mg} / \mathrm{kg})$ cocktails and placed in a stereotaxic frame (David Kopf instruments, Tujunga, CA, USA) for MSC transplantation. After a burr hole was made with a stereotaxic-attached drill, $1.2 \times 10^{5} \mathrm{hTMSCs}$ or AdMSCs $/ \mu \mathrm{L}$ in phosphate-buffered saline (PBS) were injected with a Hamilton syringe into the left lateral striatum $(0.7 \mathrm{~mm}$ anterior to the bregma, $3.2 \mathrm{~mm}$ lateral to the midline, and $5.5 \mathrm{~mm}$ beneath the dura) in a volume of $5 \mu \mathrm{L}$ for 5 minutes, and the needle was left for an additional 5 minutes after the injection. Control group was injected with PBS instead of stem cells. Subsequently, dental cement was applied on the skull and the animals were re- 
turned to their cages once they become conscious.

\section{Behavioral Assessment}

Modified neurologic severity score (mNSS), which can evaluate motor (muscle tone and abnormal movement), sensory (visual, tactile, and proprioceptive), and reflex (pinna, corneal, and startle) functions, was used for assessing the efficacy of MSC transplantation. Additionally, limb placement test was performed to check animals' proprioception and the test results were graded with mNSS (normal, 0; maximal deficit score, 27). To measure MSC-mediated functional recovery accurately, corner test was also performed, which counted the number of each turn direction in front of the corner made by 2 cardboards and calculated the left turn ratio. Tests were performed before and at 1,7 , and 14 days after MCAo surgery.

\section{Measurement of Infarct Sizes}

Infarct volume was assessed at 14 days after MCAo. After cardiac perfusion with normal saline and fixation with $4 \%$ paraformaldehyde in 0.1M PBS, the brains were removed and dehydrated with $30 \%$ sucrose solution. Then, the brains were cut into $50-\mu \mathrm{m}$-thick coronal sections by using a cryostat microtome. Total of 6 sections from each brain were processed for hematoxylin and eosin (H\&E) staining. Briefly, sections were stained in hematoxylin solution for 2 minutes, followed by washing with tap water. Then the sections were incubated with eosin for 20 seconds, dehydrated, and mounted. Using an image analysis program (Image J; Bethesda, MD, USA), infarct area was measured by subtracting the area of the noninfarcted hemisphere from that of the contralateral tissue. The percentage of infarct volume was calculated by dividing the sum of the infarct areas by the areas of contralateral hemisphere.

\section{Immunohistochemistry}

Three coronal sections from each mouse ( $450-\mu \mathrm{m}$ interval) were incubated with $10 \%$ normal donkey serum for 1 hour and then with the following primary antibodies for overnight at $4^{\circ} \mathrm{C}$ : rabbit polyclonal anti-doublecortin (anti-DCX, 1:1,000; Millipore Corp., Burlington, MA, USA), mouse monoclonal anti-human nuclei (hNU, 1:200; Millipore Corp.). After washing with PBS, the sections were incubated with the following secondary antibodies for 2 hours at room temperature $\left(22^{\circ} \mathrm{C} \pm 3^{\circ} \mathrm{C}\right)$ : Alexa488-conjugated donkey anti-mouse IgG (Jackson ImmunoResearch, West Grove, PA, USA), Cy3-conjugated donkey anti-rabbit IgG (Jackson ImmunoResearch). Then, after washing with PBS, the sections were mounted with DAPI (4, 6-diamidino-2-phenylindole)-included mounting media and observed using a confocal microscopy (LSM 510 Meta; Carl Zeiss, Oberkochen, Germany).

\section{Statistical Analysis}

The data are presented as the mean \pm standard error of the mean and statistical significance was assessed using IBM SPSS Statistics ver. 21.0 (IBM Co., Armonk, NY, USA) for behavioral tests or GraphPad Prism 7 (GraphPad Software Inc., La Jolla, CA, USA) for the rest of statistical analyses. Repeated measures analysis of variance (ANOVA) was performed for the behavioral tests, followed by Duncan post hoc test. ANOVA followed by Duncan post hoc test was performed to compare infarct volume. Student unpaired t-test was performed for immunohistochemistry for hNU and DCX as the data passed Shapiro-Wilk normality test. $\mathrm{P}<0.05$ was considered statistically significant.

\section{RESULTS}

\section{Neurologic Functional Improvement by hTMSCs}

We assessed rats' motor function at 1,7 , and 14 days after 50 minutes of MCAo surgery by using mNSS and corner test (Fig. 1A). Compared to PBS-treated rats, hTMSCs- or AdMSCstreated rats showed a significant improvement in neurologic function after ischemic stroke, evaluated by mNSS test (Fig. 1B). Moreover, corner test showed the same results over the time course of functional recovery following stroke, corroborating our mNSS findings (Fig. 1C). Collectively, we demonstrated that transplantation of hTMSCs after MCAo could show comparable therapeutic potentials with AdMSCs, of which benefits are well known against ischemic stroke.

\section{No Difference in Infarct Volume by hTMSCs}

As we found functional improvements by hTMSC administration after ischemic stroke, we performed H\&E staining for the evaluation of neuroprotective effects. When we measured the infarct size by demarcating eosinophilic dead cell areas (Fig. 2A), hTMSCs- and AdMSCs-treated groups showed the similar infarct volume compared to PBS-administered group (Fig. 2B), suggesting no neuroprotective effects by hTMSC transplantation.

\section{Survival of Grafted hTMSCs Following Ischemic Stroke}

As a next step, we evaluated the number of surviving MSCs in the brain at 14 days after ischemic stroke. Immunohistochemistry to hNU revealed many hTMSCs and AdMSCs that were la- 

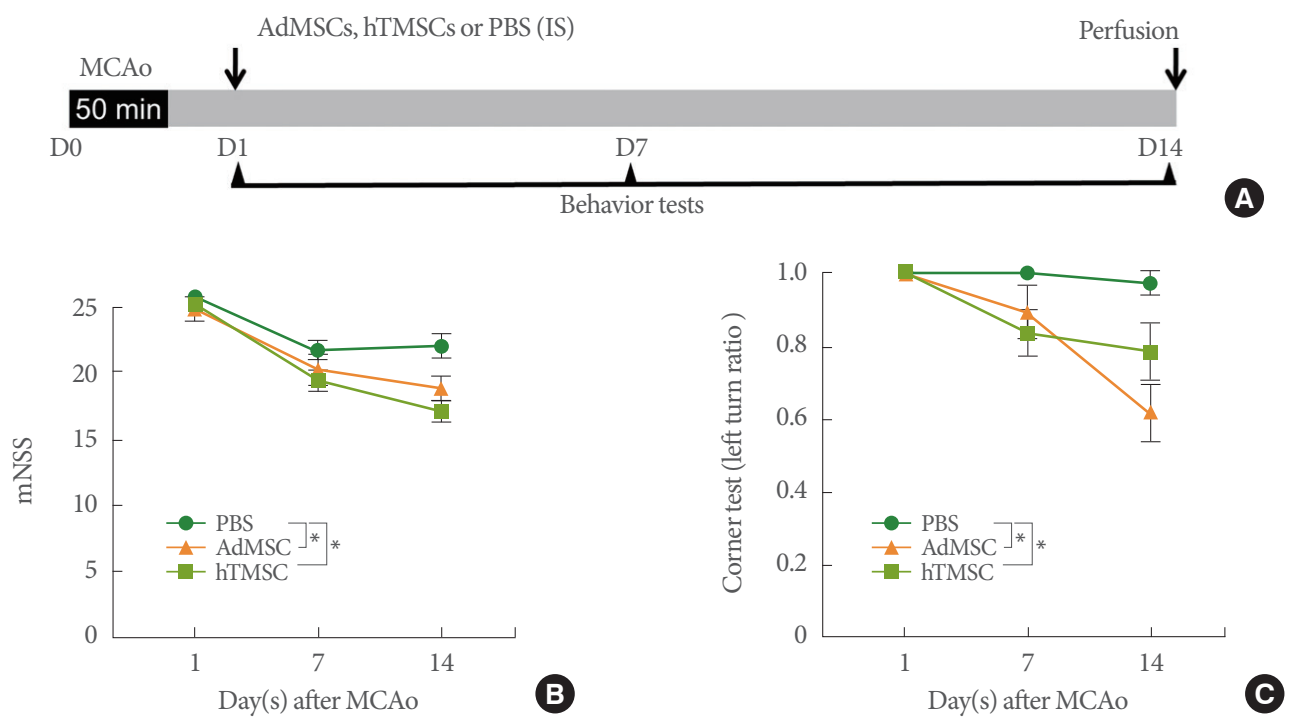

Fig. 1. Improved functional recovery following ischemic stroke. (A) Schematic illustration of intrastriatal transplantation (IS) of mesenchymal stem cells (MSCs) after intraluminal occlusion of middle cerebral artery (MCAo) and behavioral testing timeline. (B) Modified neurologic severity score (mNSS). (C) Corner test. PBS, phosphate-buffered saline; AdMSCs, adipose tissue-derived mesenchymal stem cells; hTMSCs, human turbinate-derived mesenchymal stem cells.
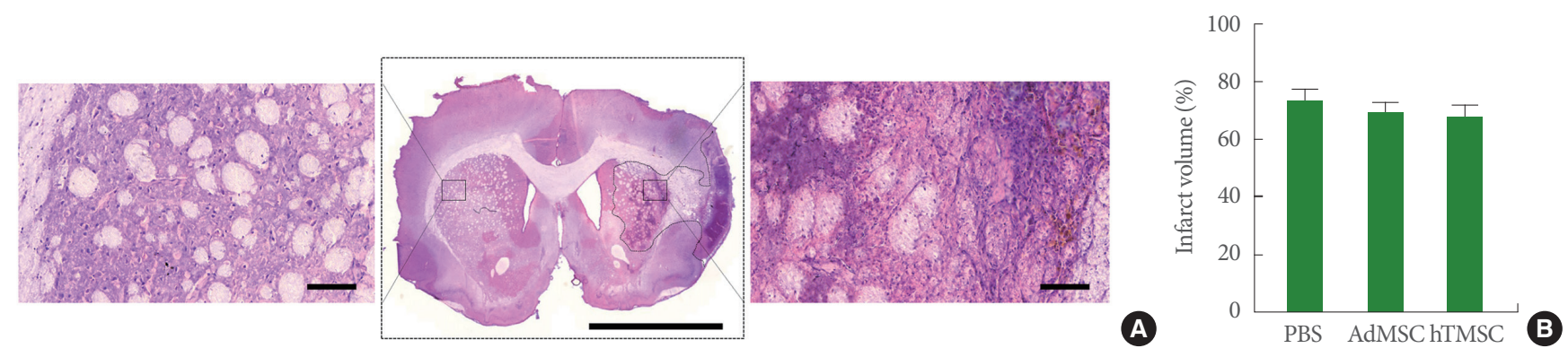

Fig. 2. Infarct volume after transplantation of adipose tissue-derived mesenchymal stem cells (AdMSCs) and human turbinate-derived mesenchymal stem cells (hTMSCs). (A) Representative images of the brain section stained with hematoxylin and eosin (H\&E). Magnified photomicrograph in the left is the contralateral striatum showing normal healthy cells. The right magnified image shows eosinophilic dead cell areas by ischemic stroke. Scale bar in the middle photomicrograph $=5 \mathrm{~mm}$, Scale bar in the left and right magnified images $=100 \mu \mathrm{m}$. (B) Percentage of infarct volume. PBS, phosphate-buffered saline.

beled with hNU in the infarct area, with a few in the penumbra (Fig. 3A). When we performed a quantitative analysis of hNUexpressing cells, we found no difference in the total number of hNU-positive cells between AdMSCs- and hTMSCs-treated brains, although there was a reducing trend in the hTMSC group (Fig. 3B). Moreover, when we separately counted the number of hNU-expressing cells in the infarct area and the penumbra, there was no difference between the 2 groups (Fig. 3B), suggesting similar survival rates of hTMSCs and AdMSCs after ischemic stroke.

\section{Increased Hippocampal Neurogenesis by hTMSCs}

In order to identify molecular mechanisms underlying benefi- cial effects of hTMSCs after ischemic stroke, we examined hippocampal neurogenesis by staining DCX (Fig. 4A). When we counted the number of DCX-expressing cells in the subgranular zone of the dentate gyrus where new neurons are constantly born, we found that hTMSCs-transplanted group showed a significant increase in the number of DCX-positive cells, compared to PBS-treated group (Fig. 4B). However, there was no difference in the number of hilar ectopic DCX-expressing cells among three groups (Fig. 4C), suggesting that ischemic injurymediated aberrant hippocampal neurogenesis was not altered by the transplantation of hTMSCs or AdMSCs. 

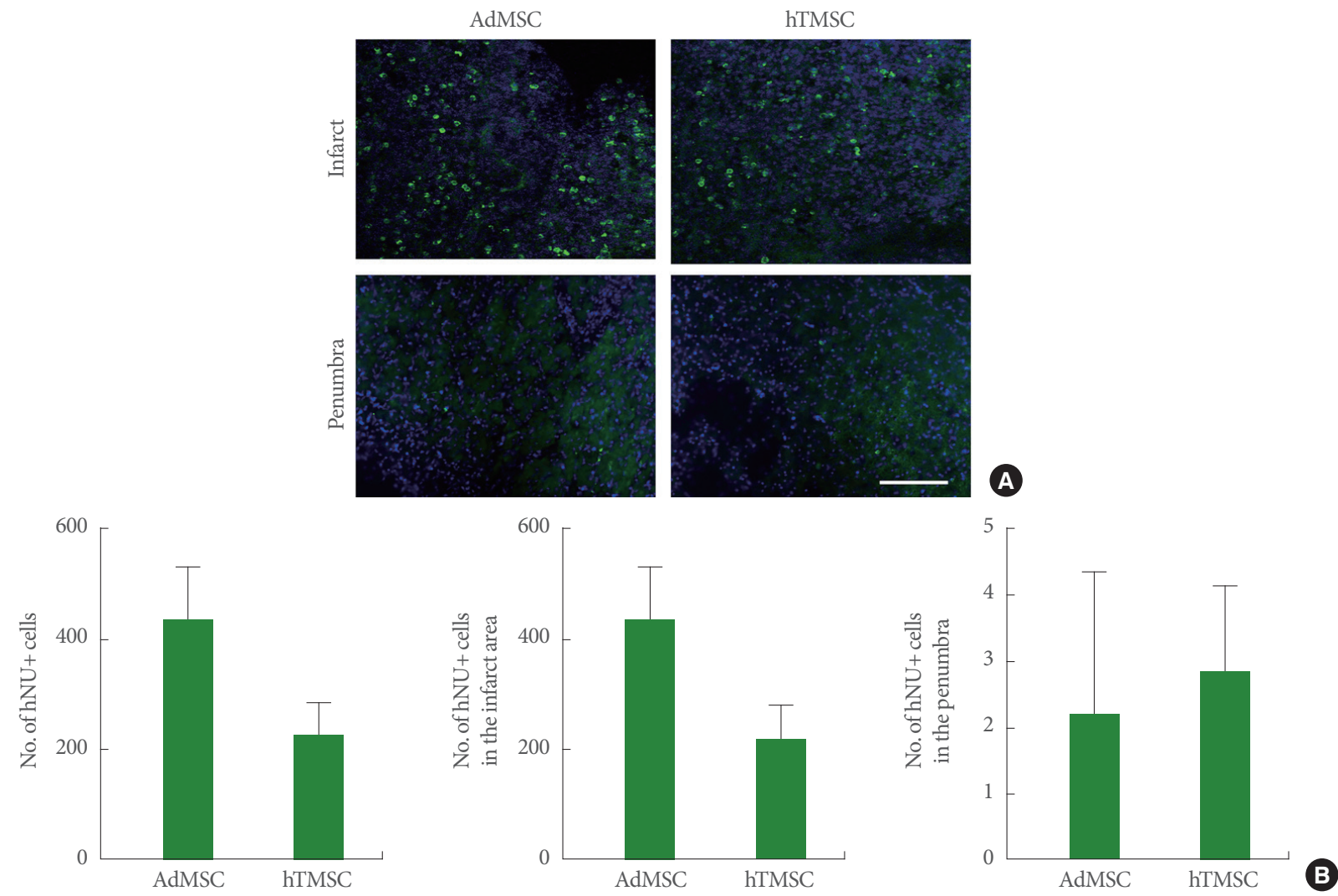

Fig. 3. Survival of grafted adipose tissue-derived mesenchymal stem cells (AdMSCs) and human turbinate-derived mesenchymal stem cells (hTMSCs). (A) Representative confocal images of human nuclei (hNU)-immunostained brain sections. Scale bar $=100 \mu \mathrm{m}$. (B) The number of hNU-expressing mesenchymal stem cells (MSCs).

\section{DISCUSSION}

In the present study, we demonstrated that MSCs isolated from the inferior turbinate (hTMSCs) could improve functional recovery following ischemic stroke. Moreover, hTMSCs showed comparable survival rates in the ischemic brain tissues compared to AdMSCs, well-known MSCs beneficial against ischemic stroke. Although transplantation of hTMSCs did not reduce the infarct size at 14 days after MCAo, administration of hTMSCs could promote the hippocampal neurogenesis, without affecting ectopic migration of newborn neurons in the dentate gyrus.

For the treatment of ischemic stroke, cell-based therapy has become a promising approach to enhance functional recovery following stroke [7,9]. Multipotent MSCs quickly emerged as attractive candidates due to their low immunogenicity and marked behavioral improvement against many neurological diseases [7]. Among various sources of MSCs, we showed for the first time that hTMSCs obtained from turbinectomy could improve behavioral outcomes after acute ischemic stroke. Intrastriatal administration of hTMSCs at 1 day after MCAo could provide comparable motor functional recoveries in 2 different neurologic examinations compared to AdMSCs, demonstrating outstanding therapeutic potentials of hTMSCs against ischemic stroke. Moreover, another study reported that hTMSCs could repair vocal fold injuries by promoting growth factors and extracellular matrix materials, supporting the excellent regenerative capabilities of hTMSCs [17]. As both hTMSCs isolated from hypertrophic and contralateral normal inferior turbinate showed indistinguishable characteristics of stem cells [13], hTMSCs we utilized in the present study are basically not pathologic, alleviating possible safety issues.

Biological mechanisms underlying the benefits of MSCs in the ischemic brain have been vigorously explored $[10,18]$. Replacement of dead brain cells with differentiated MSCs were initially considered to drive advantageous effects of MSCs in 

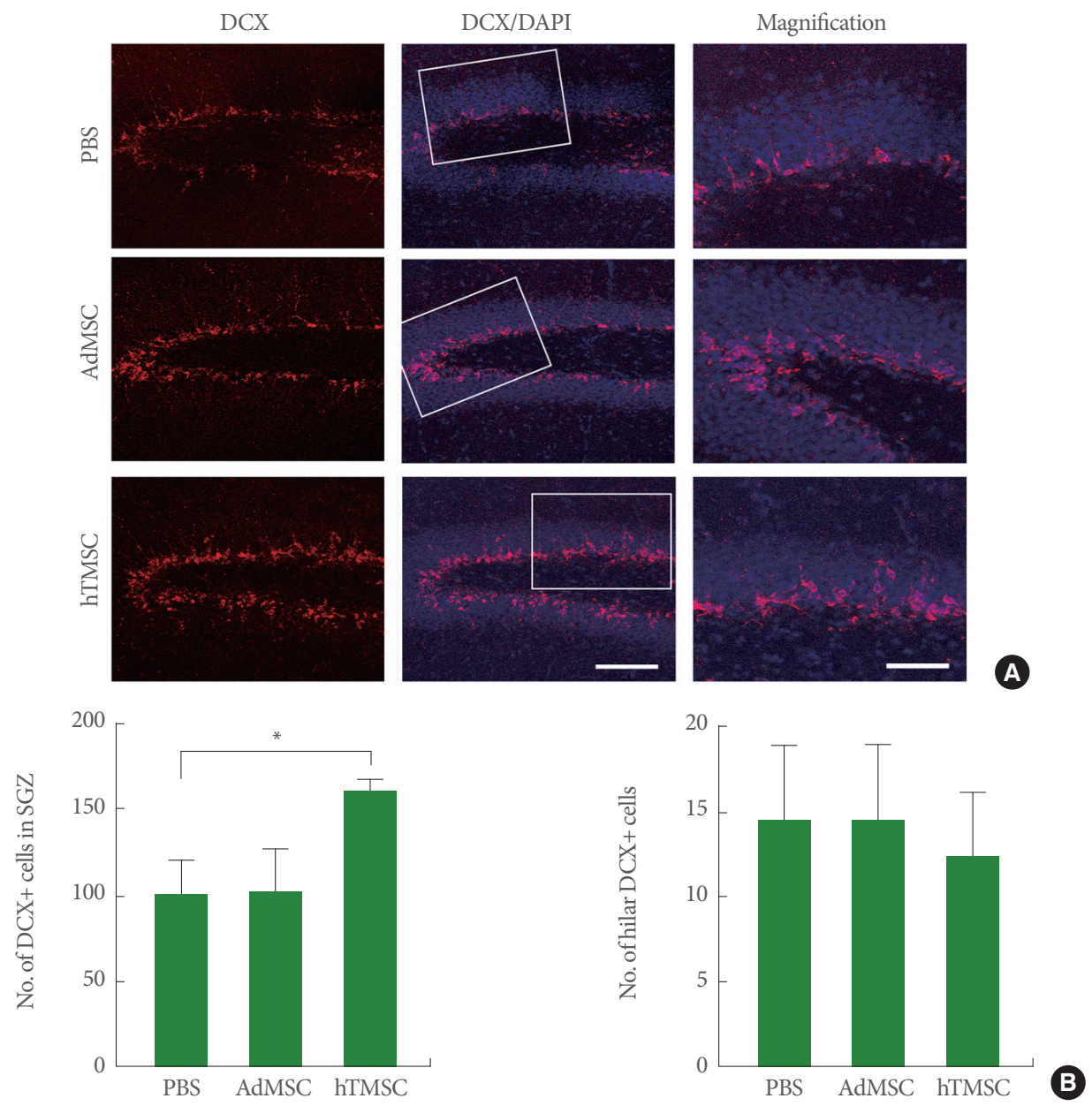

Fig. 4. Neurogenesis after transplantation of adipose tissue-derived mesenchymal stem cells (AdMSCs) and human turbinate-derived mesenchymal stem cells (hTMSCs). (A) Representative confocal images of doublecortin (DCX)-immnunostained hippocampus. White rectangles were magnified in the right column. Scale bar in the middle bottom $=100 \mu \mathrm{m}$, valid for the left and middle columns. Scale bar in the right bottom $=50 \mu \mathrm{m}$, valid for the entire right column. (B) The number of DCX-expressing cells in subgranular zone. DAPI, 4,6-diamidino-2-phenylindole; $\mathrm{PBS}$, phosphate-buffered saline. ${ }^{*} \mathrm{P}<0.05$.

ischemic stroke. However, due to extensive infarct areas for MSCs to regenerate and the very low neural differentiation rate of administered MSCs [19], other mechanisms such as the induction of angiogenesis, synapse formation, paracrine effects, or endogenous neurogenesis have been proposed for the explanations of MSC-mediated therapeutic potentials. In particular, bone marrow-derived MSC administration after MCAo could significantly increase cellular proliferation in the subventricular zone (SVZ) [20], further boosting SVZ cell proliferation following stroke. Moreover, MSCs could promote the production of newborn neurons in the brain for 2 weeks after MCAo [21]. In line with this report, we also showed the increased number of newborn neurons by hTMSC transplantation. As pharmaco- logical approaches to enhance ischemia-induced neurogenesis by erythropoietin, statin, or phosphodiesterase 5 inhibitors could improve functional outcomes after acute ischemic stroke [22], promotion of hippocampal neurogenesis can, at least partly, mediate the therapeutic efficacy of hTMSC after MCAo.

Interestingly, we found that hTMSCs could enhance hippocampal neurogenesis in the subgranular zone, but did not affect aberrantly migrating hilar DCX-expressing cells. Although it is not robust, stroke injury can generate newborn granule neurons with morphological abnormalities, i.e., cells with persistent basal dendrites, and aberrantly integrated neurons, i.e., hilar ectopic granule cells [23]. Recently, enhanced production of both dentate and ectopic granule neurons by poststroke running 
failed to improve general performance in the Morris water maze test [24], suggesting that aberrant hippocampal neurogenesis may have a significant impact on stroke-related functional impairment. Thus, our results showing the unique upregulation of normotopic neurogenesis by post-stroke hTMSCs can provide a great potential for the clinical application of hTMSCs. Further studies are required to elucidate differential regulatory mechanisms of hTMSCs on the hippocampal neurogenesis following ischemic stroke.

In conclusion, we provided a new source of MSCs that can improve neurologic function following acute ischemic stroke. Transplantation of hTMSCs showed comparable functional outcomes compared to AdMSCs, supporting excellent therapeutic effects of hTMSCs. Administration of hTMSCs promoted subgranular neurogenesis without affecting aberrant hippocampal neurogenesis, providing a scientific basis for the potential use of our newly identified MSCs for the treatment of acute ischemic stroke.

\section{AUTHOR CONTRIBUTION STATEMENT}

- Full access to all the data in the study and takes responsibility for the integrity of the data and the accuracy of the data analysis: $K O C$

- Study concept and design: $H L, S W K$

- Acquisition of data: $H L, S H P$

- Analysis and interpretation of data: $H L, S H P, S W K$

- Drafting of the manuscript: $H L, K O C$

- Critical revision of the manuscript for important intellectual content: HL, SHP, SWK

- Statistical analysis: $H L, K O C$

- Obtained funding: $K O C$

- Administrative, technical, or material support: $H L$, SHP, SWK

- Study supervision: $K O C$

\section{REFERENCES}

1. World Health Organization. The top 10 causes of death [Internet]. Geneva (Switzerland): World Health Organization; 2018. [cited 2018 Jun 20]. Available from: http://www.who.int/news-room/factsheets/detail/the-top-10-causes-of-death.

2. Albertson M, Sharma J. Stroke: current concepts. S D Med 2014; 67:455, 457-61, 463-5.

3. Powers WJ, Derdeyn CP, Biller J, Coffey CS, Hoh BL, Jauch EC, et al. 2015 American Heart Association/American Stroke Association
Focused Update of the 2013 Guidelines for the early management of patients with acute ischemic stroke regarding endovascular treatment A guideline for healthcare professionals from the American Heart Association/American Stroke Association. Stroke 2015; 46:3020-35.

4. Kurozumi K, Nakamura K, Tamiya T, Kawano Y, Ishii K, Kobune M, et al. Mesenchymal stem cells that produce neurotrophic factors reduce ischemic damage in the rat middle cerebral artery occlusion model. Mol Ther 2005;11:96-104.

5. Bang OY. Clinical trials of adult stem cell therapy in patients with ischemic stroke. J Clin Neurol 2016;12:14-20.

6. Kalladka D, Sinden J, Pollock K, Haig C, McLean J, Smith W, et al. Human neural stem cells in patients with chronic ischaemic stroke (PISCES): a phase 1, first-in-man study. Lancet 2016;388:787-96.

7. Hsuan YC, Lin CH, Chang CP, Lin MT. Mesenchymal stem cellbased treatments for stroke, neural trauma, and heat stroke. Brain Behav 2016;6:e00526.

8. Castillo M, Liu K, Bonilla L, Rameshwar P. The immune properties of mesenchymal stem cells. Int J Biomed Sci 2007;3:76-80.

9. Doeppner TR, Hermann DM. Mesenchymal stem cells in the treatment of ischemic stroke: progress and possibilities. Stem Cells Cloning 2010;3:157-63.

10. Chopp M, Li Y. Treatment of neural injury with marrow stromal cells. Lancet Neurol 2002;1:92-100.

11. Li Y, Chen J, Wang L, Lu M, Chopp M. Treatment of stroke in rat with intracarotid administration of marrow stromal cells. Neurology 2001;56:1666-72.

12. Hwang SH, Kim SY, Park SH, Choi MY, Kang HW, Seol YJ, et al. Human inferior turbinate: an alternative tissue source of multipotent mesenchymal stromal cells. Otolaryngol Head Neck Surg 2012;147:568-74.

13. Hwang SH, Park SH, Choi J, Lee DC, Oh JH, Kim SW, et al. Characteristics of mesenchymal stem cells originating from the bilateral inferior turbinate in humans with nasal septal deviation. PLoS One 2014;9:e100219.

14. Kim SW, Cho JH, Hong MW, Rhie JW, Yoon HR. Induction of chondrogenic differentiation in cultured fibroblasts isolated from the inferior turbinate. Otolaryngol Head Neck Surg 2008;139:1438.

15. Chang KA, Lee JH, Suh YH. Therapeutic potential of human adipose-derived stem cells in neurological disorders. J Pharmacol Sci 2014;126:293-301.

16. Cho KO, Kim YS, Cho YJ, Kim SY. Upregulation of DSCR1 (RCAN1 or Adapt78) in the peri-infarct cortex after experimental stroke. Exp Neurol 2008;212:85-92. 
17. Kim CS, Choi H, Park KC, Kim SW, Sun DI. The ability of human nasal inferior turbinate-derived mesenchymal stem cells to repair vocal fold injuries. Otolaryngol Head Neck Surg 2018;159:335-42.

18. Honmou O, Onodera R, Sasaki M, Waxman SG, Kocsis JD. Mesenchymal stem cells: therapeutic outlook for stroke. Trends Mol Med 2012;18:292-7.

19. Chen X, Li Y, Wang L, Katakowski M, Zhang L, Chen J, et al. Ischemic rat brain extracts induce human marrow stromal cell growth factor production. Neuropathology 2002;22:275-9.

20. Chen J, Li Y, Katakowski M, Chen X, Wang L, Lu D, et al. Intravenous bone marrow stromal cell therapy reduces apoptosis and promotes endogenous cell proliferation after stroke in female rat. J Neurosci Res 2003;73:778-86.

21. Yoo SW, Kim SS, Lee SY, Lee HS, Kim HS, Lee YD, et al. Mesen- chymal stem cells promote proliferation of endogenous neural stem cells and survival of newborn cells in a rat stroke model. Exp Mol Med 2008;40:387-97.

22. Zhang ZG, Chopp M. Neurorestorative therapies for stroke: underlying mechanisms and translation to the clinic. Lancet Neurol 2009;8:491-500.

23. Niv F, Keiner S, Krishna K, Witte OW, Lie DC, Redecker C. Aberrant neurogenesis after stroke: a retroviral cell labeling study. Stroke 2012;43:2468-75.

24. Woitke F, Ceanga M, Rudolph M, Niv F, Witte OW, Redecker C, et al. Adult hippocampal neurogenesis poststroke: more new granule cells but aberrant morphology and impaired spatial memory. PLoS One 2017;12:e0183463. 